\title{
The calibration of vehicle and pedestrian flow in Mangalore city using PARAMICS
}

\author{
S. K. Prusty, R. Phadnis \& Kunal \\ National Institute Of Technology Karnataka, India
}

\begin{abstract}
This paper presents a traffic simulation approach for evaluating the pedestrian behaviour by developing a model which includes pedestrians in a vehicular micro-simulation model i.e. PARAMICS that provides a default mechanism for simulating pedestrian movements. This approach is demonstrated by using a case study of a signalized intersection having large pedestrian flow interacting with large vehicular flow in the city of Mangalore, India. In this paper pedestrian behaviour is calibrated with pedestrian speed flow models by defining pedestrians as vehicles. This work provides different methods for evaluating policies that effect both pedestrian and vehicle flow. In this work different critical factors that affect the pedestrian and vehicle flow are considered (i.e. Vehicle queue discharge rate, Pedestrian queue discharge rate, Pedestrian gap acceptance, Pedestrian speed etc.) and finally the network was coded in PARAMICS. The major parameters defined of the driving behaviour that control the car-following behaviour, lane changing behaviour, lateral position and driver reaction to traffic lights are considered and calibrated in PARAMICS. After calibration and validation of pedestrian and vehicle flows the difference between the simulated and observed data were analysed. The high variability in data sets confines the simulation resulting in a constant queue discharge rate at flows approaching saturated conditions. This work finally concludes that PARAMIC'S car-following algorithm is very much helpful in reproducing vehicle and pedestrian flow in complex as well as heterogeneous urban traffic. Application of this car-following algorithm demonstrated by calibrating in PARAMICS will be very useful in predicting known pedestrian speed-flow relationship.

Keywords: pedestrian, pedestrian speed-flow, travel time, queue discharge rate, vehicle flow, traffic flow, car-following, PARAMICS, calibration, validation.
\end{abstract}




\section{Introduction}

Since the last decades' research of pedestrian traffic flows has only been given limited attention till now, visualizing and analysing pedestrian flow is very important. In this paper a traffic simulation model is developed in which simulation of individual pedestrian and their interactions with the vehicular traffic in a network of urban roads and pedestrian crossings. The paper includes both pedestrian and vehicle flow by Ishaque and Noland [1], so it is important to analyse the real world condition of both as close as possible. This model is developed by considering pedestrian as vehicle and setting various parameters to represent the pedestrian behaviour using PARAMICS. The model is then calibrated and validated by comparing data from an actual network of Mangalore City where the pedestrian and vehicle interaction is more.

In this paper the pedestrian level of service is calculated using Fruin [2] which is predominantly for city streets. The model is developed especially to enhance the complex interaction between pedestrians and vehicles. This study indicates the importance of additional factors (i.e., Pedestrian queue discharge rate, Pedestrian gap acceptance, Pedestrian speed) which is to be related with the vehicle flow. It also shows the effort to better understand pedestrian level of service under various conditions. In fact, the primary goal is to find models which are as simple as possible, but at the same time could reflect realistic behaviours.

\section{Data collection}

The research site selected for this paper is a signalized intersection having large number of pedestrian and vehicular flow interaction, which is decided based on the pedestrian flow data from Mangalore Urban Development Authority. The signalized intersections at Ambedkar circle and Hampankatta circle and unsignalized intersection at Bunts circle of Mangalore city were considered. There are multiple lanes which can be used by all types of vehicles. The speed limit is $50 \mathrm{~km} / \mathrm{hr}$.

The area considered forms an important interchange for public transport, commercial complexes, hospitals, hotels and Cineplex. Two major bus stops are present within the study area i.e. one near Ambedkar circle and the other near Hampankatta circle. Figure 1 shows the study area considered in this paper.

\subsection{Flows and vehicle travel times}

Apart from vehicular and pedestrian interaction, vehicular traffic may also be in conflict with pedestrian crossing at the intersection. Data collection from the site for both vehicle and pedestrian traffic is very important in order to represent traffic flow in micro simulation. Data on vehicle travel time, pedestrian flow, vehicle flow were simultaneously collected at three locations $1300 \mathrm{~m}$ and two 
signalized intersections for two days in Jan, 2014. One of the two days is weekday and the other is Sunday. Data were collected in those locations using two video cameras each in every location.

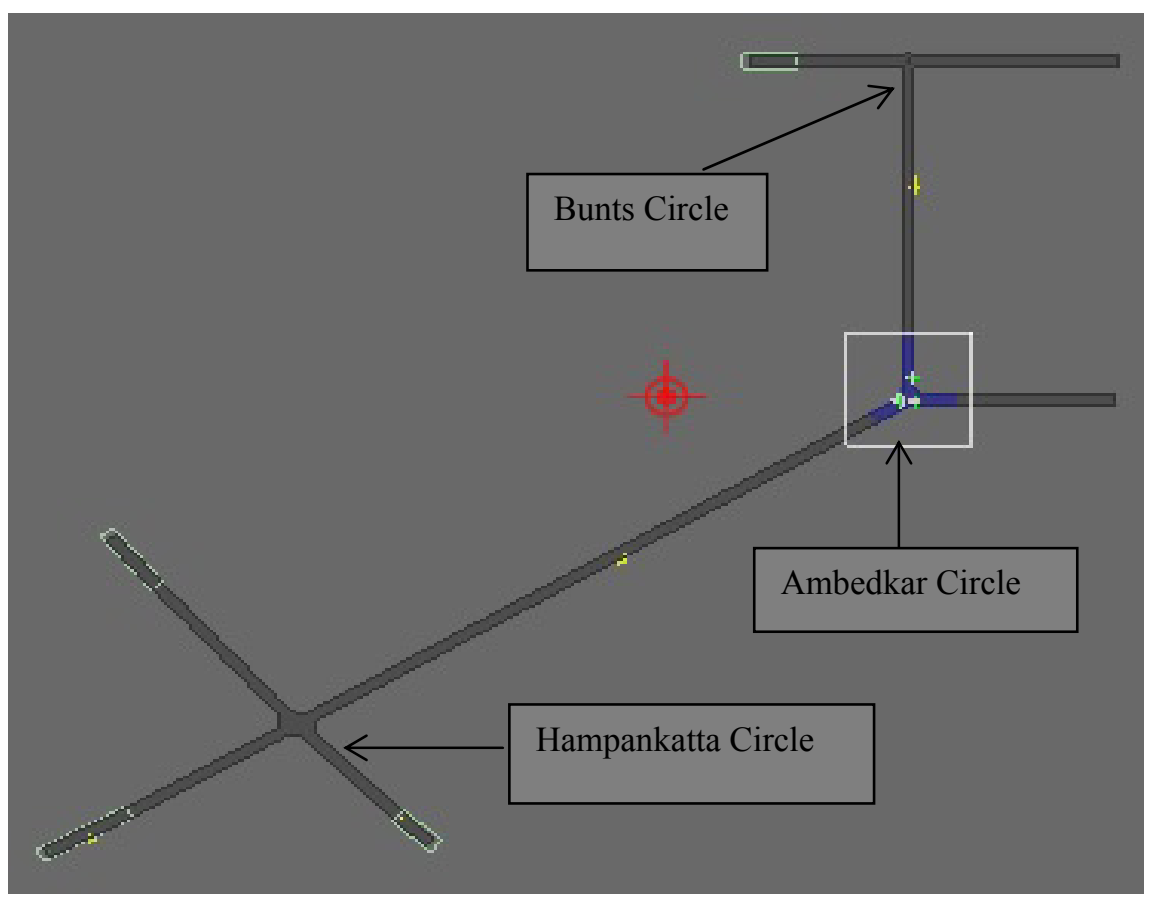

Figure 1: Road network as coded in PARAMICS.

As in India it is a mixed traffic condition, since heterogeneous or mixed traffic systems operate very differently, compared to homogeneous traffic systems. The traffic in mixed flow is comprised of fast moving and slow moving vehicles or motorized and non-motorized vehicles. The vehicles also vary in size, maneuverability, control and dynamic characteristics. Traffic is not segregated by vehicle type and therefore, all vehicles travel in the same right of way. Smaller size vehicles often squeeze through any available gap between large size vehicles and move in a haphazard manner, therefore cameras were focused on each and every vehicle number plates. The vehicle flow on Bunts Circle and Hampankatta Circle for both weekday and Sunday is shown in Table 1.

Table 1: Vehicle flows per hour for through traffic.

\begin{tabular}{|c|c|c|}
\hline Date & $\begin{array}{c}\text { Bunts Circle } \\
\text { Intersection }\end{array}$ & $\begin{array}{c}\text { Hampankatta Circle } \\
\text { Intersection }\end{array}$ \\
\hline Sun, Feb 09 2014 & 1864 & 2047 \\
\hline Mon, Feb 10 2014 & 1971 & 2139 \\
\hline
\end{tabular}


Pedestrians moving in opposing directions on the pedestrian crossing separately. The pedestrian flow data is enlisted in Table 2.

Table 2: Hourly pedestrian flows at Ambedkar and Hampankatta Circle intersections for two days.

\begin{tabular}{|c|c|c|c|c|c|c|}
\hline \multirow{2}{*}{ Date } & \multicolumn{3}{|c|}{$\begin{array}{c}\text { Ambedkar Circle } \\
\text { Intersection }\end{array}$} & \multicolumn{3}{c|}{$\begin{array}{c}\text { Hampankatta Circle } \\
\text { Intersection }\end{array}$} \\
\cline { 2 - 7 } & $\begin{array}{c}\text { South } \\
\text { bound }\end{array}$ & $\begin{array}{c}\text { North } \\
\text { bound }\end{array}$ & Total & $\begin{array}{c}\text { South } \\
\text { bound }\end{array}$ & $\begin{array}{c}\text { North } \\
\text { bound }\end{array}$ & Total \\
\hline Sun, Feb 09 2014 & 297 & 231 & 528 & 279 & 361 & 640 \\
\hline Mon, Feb 10 2014 & 317 & 269 & 586 & 457 & 612 & 1109 \\
\hline
\end{tabular}

The traffic composition was observed from the video recordings and is listed in Table 3.

Table 3: Hourly vehicle composition of through traffic at Bunts circle intersection.

\begin{tabular}{|c|c|c|c|c|}
\hline Date & 2-Wheeler & 3-Wheeler & 4-Wheeler & Bus \\
\hline Sun, Feb 09 2014 & 899 & 255 & 544 & 166 \\
\hline Mon, Feb 10 2014 & 951 & 269 & 575 & 176 \\
\hline
\end{tabular}

Vehicle composition was matched from each camera and the travel time is calculated. The effective travel time taken by the vehicle is shown Table 4 .

Table 4: Vehicle counts for which travel times were measured.

\begin{tabular}{|c|c|c|c|}
\hline Date & $\begin{array}{c}\text { Number of } \\
\text { Vehicles } \\
\text { Captured }\end{array}$ & $\begin{array}{c}\text { Capture Duration } \\
\text { (min) }\end{array}$ & $\begin{array}{c}\text { Volume Capture } \\
(\%)\end{array}$ \\
\hline Sun, Feb 09 2014 & 278 & 60 & 13 \\
\hline Mon, Feb 10 2014 & 331 & 60 & 15 \\
\hline
\end{tabular}

\subsection{Road geometry}

Road geometric data was obtained from field measurement. Specific road features required for modelling, such as location and width of pedestrian crossings, width and length of central medians at staggered crossings, vehicle stop lines, and location of bus stops were also obtained with field measurements. 
The road network was then plotted in PARAMICS considering all the road features and keeping map obtained from Google map on the background as layout.

\subsection{Signal timings}

As the signal control system of Mangalore city works under pre-time system (PTS), signal timings were obtained from site observation from study area and considered constant parameters for signal timings in PARAMICS. Signal timing of 120 second red time, pedestrian signal of 18 seconds and 40 seconds of green time is considered for signal design in PARAMICS.

\subsection{Vehicle queue discharge rate}

Determining the saturation flow rate can be a somewhat complicated matter. The saturation flow rate depends on roadway and traffic conditions. The saturation flow rate is a fundamental parameter to measure the intersection capacity and time the signalized intersections. It is also important that the vehicle is not affected by any blocks in the downstream from the position they are starting. The saturation flow rate is normally given in terms of straight-through passenger cars per hour of green. Vehicle headways were then measured between the fifth and tenth vehicle leaving the stop line. Vehicle headway obtained from the observations is $2.17 \mathrm{sec}$. Based on this vehicle headway value; saturation flow rate calculated using the procedure given by Turner and Harahap [3] is 1659 PCU per lane per hour.

\subsection{Pedestrian speeds}

The most relevant parameter that can have a direct impact on the speed results of pedestrians is the desired speed distribution as studied by Willis et al. [4]. From the video obtained from site pedestrian free flow speed has taken into considerations. The pedestrians not moving in a group, not carrying any goods, pedestrians not standing at the crossing are mainly considered. Pedestrian travel time is measured, at the instant pedestrian stepping off at the first curb to stepping onto the opposite curb. The length of the pedestrian crossing was $14 \mathrm{~m}$ onsite. The sample collected is not mentioned in separate age groups. The disaggregated result of pedestrian flow is shown in Table 5.

Table 5: Pedestrian speed statistics.

\begin{tabular}{|l|c|c|c|}
\hline Gender & Sample Size & $\begin{array}{c}\text { Mean Speed } \\
(\mathrm{km} / \mathrm{hr})\end{array}$ & $\begin{array}{c}\text { Standard } \\
\text { Deviation }\end{array}$ \\
\hline Male & 182 & 4.58 & 0.4097 \\
\hline Female & 151 & 4.25 & 0.3387 \\
\hline
\end{tabular}




\subsection{Pedestrian queue discharge rate}

The video taken on the weekday is considered for determining pedestrian queue discharge rate. The pedestrian queue discharge was observed as the time difference between when the first person in a waiting platoon started walking and when the last person in the platoon started walking. Pedestrians joining later to the last person walking in the platoon are not considered. Regressional analysis was observed from the pedestrian queue discharge rate data.

\subsection{Pedestrian gap acceptance}

The pedestrian's behaviour relating to signals is a very important factor to be considered. This pedestrian behavioural analysis is related to the vehicular flow as well as is much dependent on the pedestrian gap acceptance. As the intersection had large number of vehicular flow, the pedestrian gap was observed very less during the green phase of the vehicles only rarely were pedestrians able to cross the road against a red light. Pedestrian gap acceptance analysed only the crossings during the pedestrian clearance interval. A gap was measured as the difference between the time a pedestrian started to cross and the time when the leading vehicle in the vehicle platoon reached the pedestrian crossing. These gap readings were observed for both weekday and Sunday.

\section{Network coding}

The road network was coded in PARAMICS considering all the road features and keeping map obtained from Google maps on the background as layout. These features included such as location and width of pedestrian crossings, width and length of central medians at staggered crossings, vehicle stop lines, and location of bus stops. All major and minor streets crossing or joining the network is also coded in PARAMICS.

Pedestrian origins and destinations were coded so that pedestrians either walked only along or across Ambedkar circle. More detailed observations along Ambedkar circle were conducted to verify the pedestrian movements. As our main aim is to study the interaction between pedestrians and vehicles and validate this in PARAMICS, we are not concerned with actual destinations.

Dimensions for buses, cars, 2-wheeler, and 3-wheeler were obtained from the data obtained from Mangalore Urban Development Authority as well as through field observations. Vehicle desired speed were also considered while network coding and their speed distribution are shown in Table 6.

Table 6: Percentage distribution of vehicle speeds.

\begin{tabular}{|c|c|c|c|c|}
\hline $\begin{array}{c}\text { Speed Range } \\
(\mathrm{k} / \mathrm{mph})\end{array}$ & 2-Wheeler & 3-Wheeler & 4-Wheeler & Bus \\
\hline$<30$ & 51 & 63 & 47 & 69 \\
\hline $30-45$ & 37 & 29 & 38 & 27 \\
\hline $45-60$ & 12 & 08 & 10 & 04 \\
\hline$>60$ & 00 & 00 & 05 & 00 \\
\hline
\end{tabular}




\section{Calibration of vehicle flow}

The data collected on weekday is calibrated using PARAMICS. The driving behaviour in PARAMICS is defined by a number of parameters that control the car-following behaviour, lateral position, lane-changing behaviour, and driver reaction to traffic lights. Most microscopic traffic simulation models have the property to represent random variations in the behaviour of the simulated traffic. In microscopic models this randomness, is used in many of the simulated processes. Since the simulation has these random processes as components, several runs are required to generate valid predictions. Therefore, for this project we have run every simulation scenario 20 times. Our simulations have duration of 1 real hour for each run. From the weekday data travel time graph is plotted considering both observed and simulated travel time for each category of vehicle. This graph is shown in Figure 2.

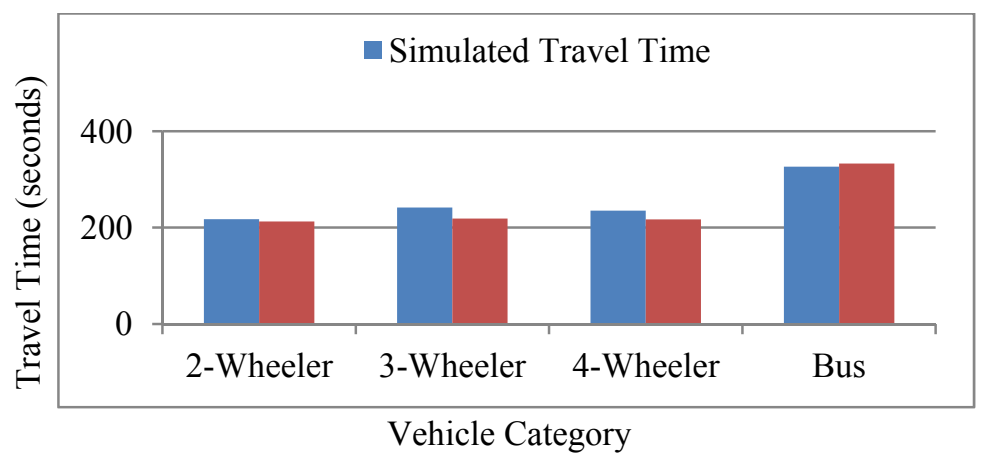

Figure 2: Travel time plots from multiple PARAMICS runs and weekday data.

For a better understanding of vehicle flow one more graph representing both observed and simulated speed for each category of vehicle is plotted using the weekday data shown in Figure 3.

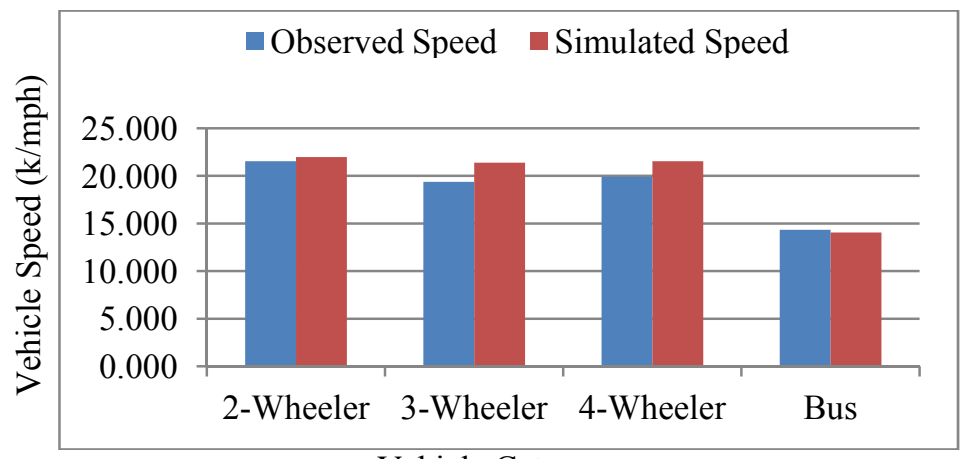

Vehicle Category

Figure 3: Travel speed plots from multiple PARAMICS runs and weekday data. 
The result for statistical correlation obtained using Pearson's Correlation Coefficient shows a very strong relation between the PARAMICS runs and weekday field data. The statistical correlation results are shown in Table 7.

Table 7: Correlation between mean of field travel time data of weekday and 10 PARAMICS run Model Using Pearson's Correlation Coefficient.

\begin{tabular}{|l|l|l|}
\hline & PARAMICS Run & Field Data \\
\hline Correlation Coefficient & 1.00 & 0.91 \\
\hline Significance (2-tailed) & & 0.00 \\
\hline N & 19 & 19 \\
\hline
\end{tabular}

\section{Validation of vehicle flows}

The travel time data obtained from Sunday is used for validation of vehicle flows. The major difference observed between Sunday data and weekday data was traffic composition. Data collected on Sunday was having low proportion of 2-wheeler, 3-wheeler, 4-wheeler and buses as compared to the weekday data. Therefore, Sunday data has been considered separately and been used for validation purpose. The travel time graph shown in Figure 4 indicates both observed and simulated travel time obtained from Sunday data.

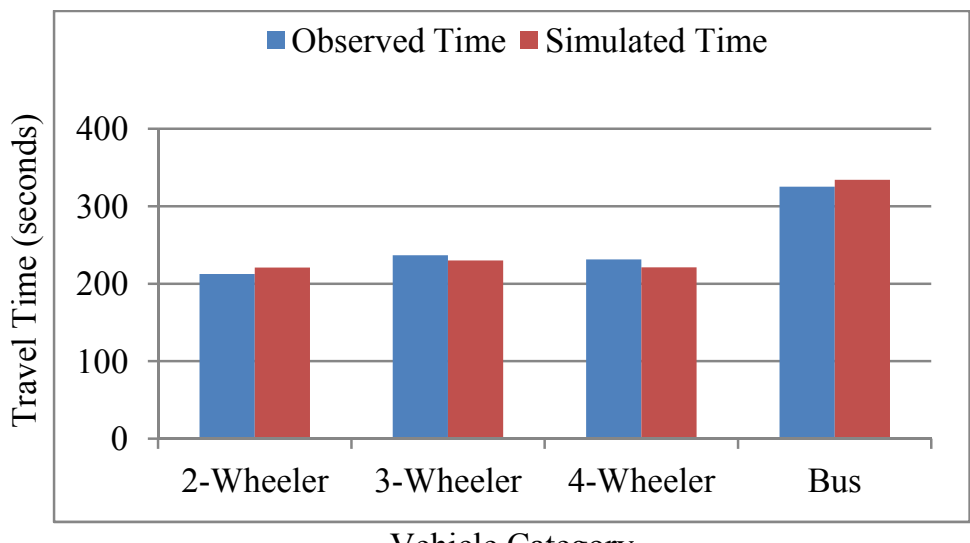

Vehicle Category

Figure 4: Vehicle travel time plots from multiple PARAMICS runs and Sunday data.

As there are differences in travel time between weekday and Sunday data, a graph is plotted between observed and simulated vehicle travel speed for Sunday data which is shown in Figure 5 for better understanding of traffic behaviour. 


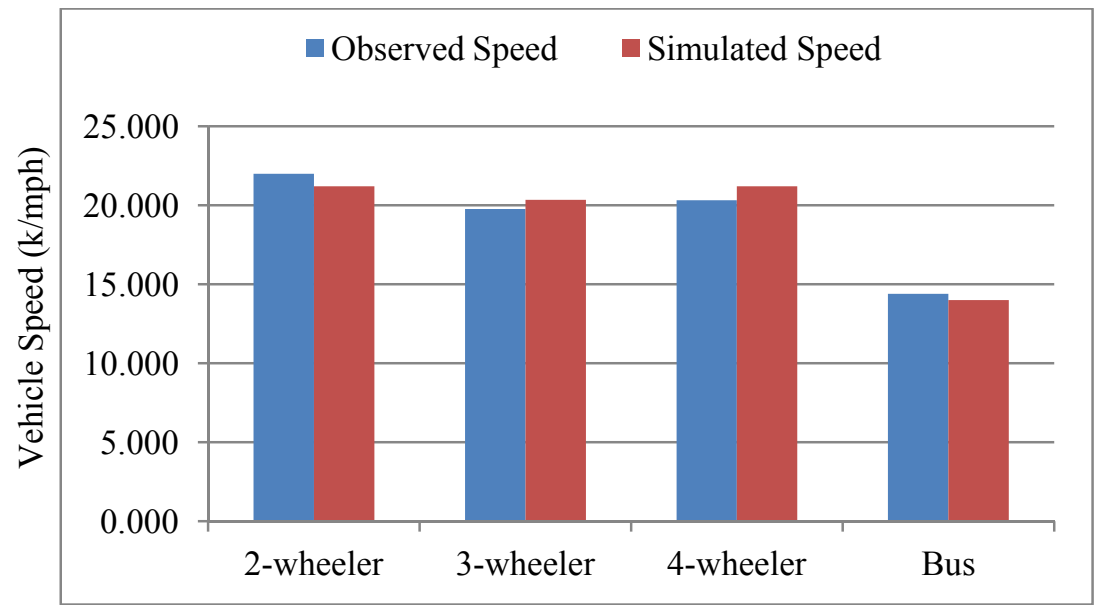

Vehicle Category

Figure 5: Vehicle travel speed plot from multiple PARAMICS runs and Sunday data.

These differences were not a cause of concern due to the expected day-to-day variation in the time ranges. Sacks et al. [5] shows statistical tests were performed for validation same as performed for calibration of vehicle flows, to check correlations and significance of the difference in means. A strong correlation coefficient between the mean of 10 PARAMICS run and the Sunday data was found and shown in Table 8 .

Table 8: Correlation between mean of field travel time data of Sunday and 10 PARAMICS run Model Using Pearson's Correlation Coefficient.

\begin{tabular}{|l|l|l|}
\hline & PARAMICS Run & Field Data \\
\hline Correlation Coefficient & 1.00 & 0.93 \\
\hline Significance (2-tailed) & & 0.00 \\
\hline $\mathrm{N}$ & 10 & 10 \\
\hline
\end{tabular}

\section{Calibration of pedestrian flows}

According to the existing literatures, pedestrian simulation models mainly include two categories: discrete models and continuous models. Continuous models are based on pedestrian flow dynamics, which describe pedestrian moving behaviour by establishing continuous function according to pedestrian behaviour characteristics.

In this study, pedestrian flow speed is the average value of $25 \%$ samples among all pedestrians. Therefore, the parameter Calibration in this study only aims at social force model developed by Helbing and Molnar [6]. Calibration of pedestrian flows in PARAMICS was performed on a $100 \mathrm{~m}$ long simple network consisting of one path. Pedestrian walkway of two widths of $1 \mathrm{~m}$ and $2 \mathrm{~m}$ were 
analysed for the $100 \mathrm{~m}$ long pedestrian path. Pedestrian size and parameters in the vehicle-following model of PARAMICS were altered to result in a close match to Fruin's speed-flow model for bidirectional pedestrian movements. The pedestrian speed flow curve for different widths against Fruins bidirectional model is shown in Figure 6. Fritzsche car following model is used for selecting parameters for pedestrian modelling in PARAMICS and the parameters are listed in Table 9.

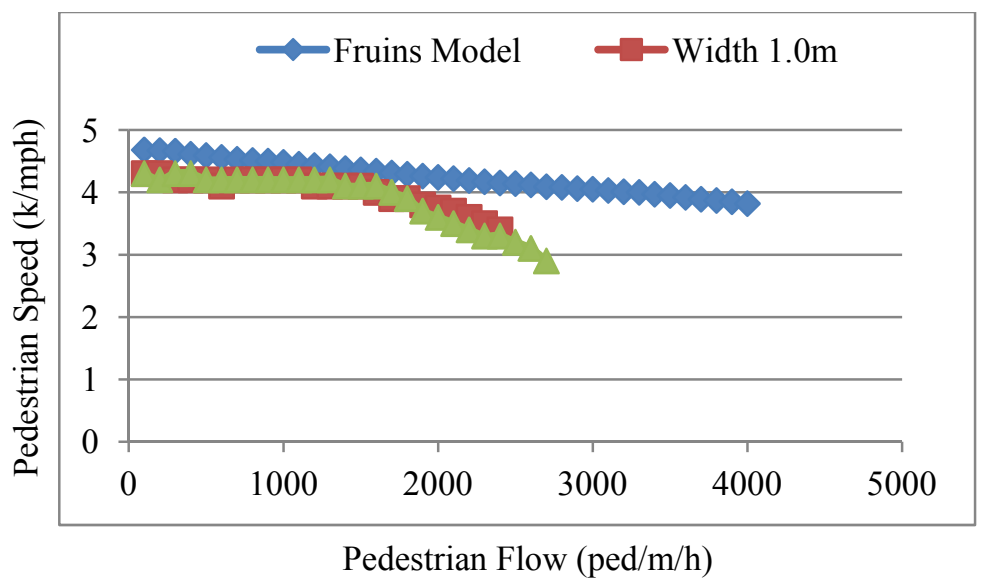

Figure 6: Pedestrian speed-flow relationship with the results of calibrated PARAMICS model plotted against Fruins models.

Table 9: Parameters values used in PARAMICS.

\begin{tabular}{|l|l|c|}
\hline Parameters & & $\begin{array}{l}\text { Values } \\
\text { Used }\end{array}$ \\
\hline Car following model & Fritzsche (PARAMICS) & \\
\hline Model parameters & Average standstill distance (m) & 1.0 \\
\hline Look-ahead distance & Minimum (m) & 1.0 \\
\cline { 2 - 3 } & Maximum (m) & 3.0 \\
\hline Minimum headway & Front/rear(m) & 0.01 \\
\hline $\begin{array}{l}\text { Overtake on same } \\
\text { lane }\end{array}$ & & ON \\
\hline $\begin{array}{l}\text { Pedestrian } \\
\text { dimensions }\end{array}$ & length (m) & 0.37 \\
\cline { 2 - 3 } & width (m) & 0.20 \\
\hline
\end{tabular}

\section{Validation of pedestrian flows}

The pedestrian speed flow data collected from Ambedkar Circle is further introduced into PARAMICS and calibrated for Fruins model which resulted the correlation coefficient of 0.84 . As the correlation coefficient value was feasible for accommodating higher number of pedestrian flow, recalibration was not 
needed. The parameters used for calibrating the pedestrian model using Fruins model were left unchanged for pedestrian flow on the sidewalk as high pedestrian densities were present at crossing compared to the sidewalks. Pedestrian travel time obtained from Sunday field data and PARAMICS run is shown in Figure 7.

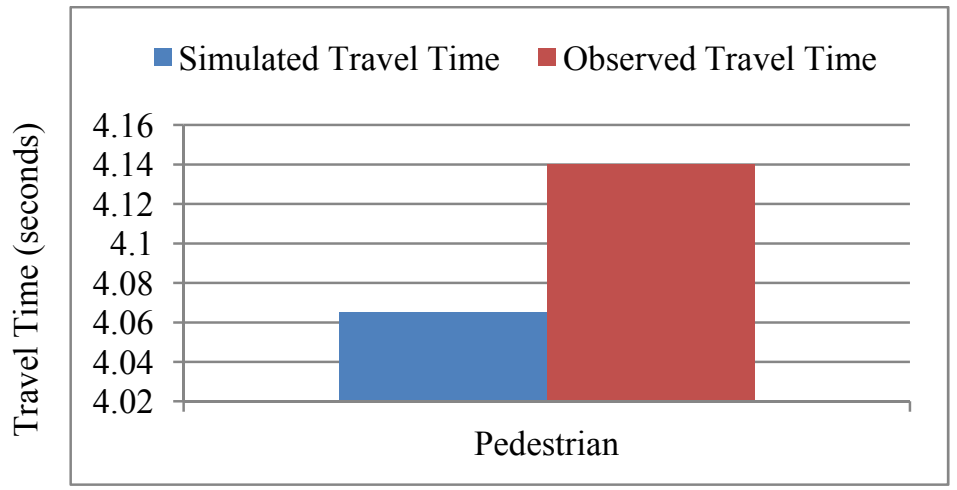

Figure 7: Pedestrian travel time plot from multiple PARAMICS runs and Sunday data.

To explicitly define the validation of pedestrian flow, a graph plotted between observed and simulated pedestrian travel speed obtained from Sunday data is shown in Figure 8.

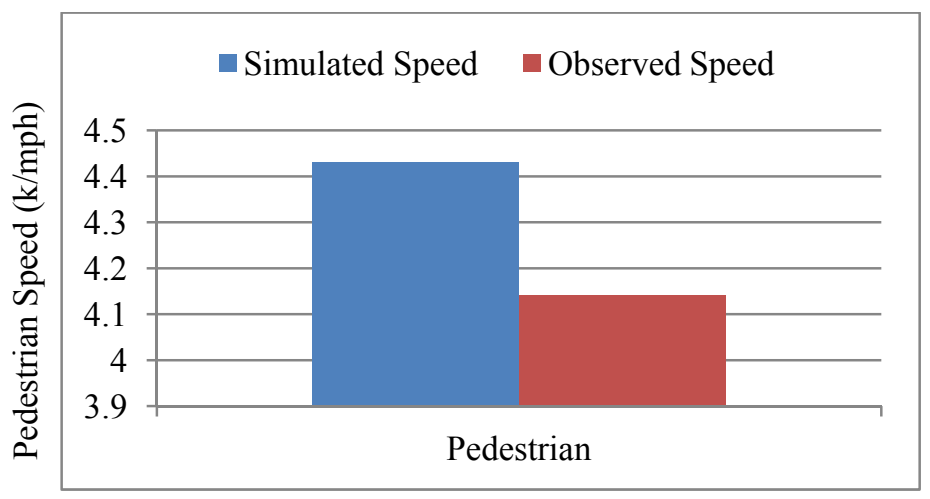

Figure 8: Pedestrian travel speed plot from multiple PARAMICS runs and Sunday data.

\section{Conclusions}

The calibration and validation of the vehicle-pedestrian traffic model concludes that PARAMIC'S car-following algorithm is very much helpful in reproducing vehicle and pedestrian flow in mixed or heterogeneous urban traffic condition. 
Many works have been done based on pedestrian flow, but using car-following theory for pedestrian modelling is very different. Fritzsche car-following model is used in this paper for pedestrian modelling. Application of this car-following algorithm demonstrated by calibrating in PARAMICS will be very useful in predicting known pedestrian speed-flow relationship.

The graph between observed and simulated vehicle travel time obtained from validation of vehicle flow shows that there is no significant difference. For the calibration of pedestrian flows, speed flow relationship is plotted for different widths using multiple PARAMICS runs against Fruins model.

In this paper Pearson's correlation coefficient is used to correlate the field data and simulated data for both weekday and Sunday. The correlation coefficient value obtained during validation of pedestrian flow is more than 0.8 shows the feasibility for large number of pedestrian flow and also satisfies the main objective of the study. There are a number of issues that could be studied further in this field.

\section{References}

[1] Ishaque, M.M. and Noland, R.B. (2009). "Pedestrian and vehicle flow calibration in multimodal traffic simulation." J. Transp. Eng., ASCE, 135:338-348.

[2] Fruin, J. J. (1971). "Designing for pedestrians: A level of service concept." Highway Res. Rec., 355, 1-15.

[3] Turner, J., and Harahap, G. (1993). "Simplified saturation flow data collection methods." Transport Research Laboratory, Rep. PA1292/93, Crowthorne, U.K.

[4] Willis, A., Gjersoe, N., Havard, C., Kerridge, J., and Kukla, R. (2004). "Human movement behaviour in urban spaces: Implications for the design, and modelling of effective pedestrian environments." Environ. Plan. B: Plan. Des. 31, 805-828.

[5] Sacks, J., Rouphail, N., Park, B., and Thakuriah, P. (2002). "Statistically based validation of computer simulation models in traffic operations, and management." J. Transp. Stat., 5(1). 1-24.

[6] Helbing, D., and Molnar, P. (1995). "Social force model of pedestrian dynamics." Phys. Rev. E, 51, 4282-4286.

[7] Hochberg, Y. and Tamhane, A. C. (1987). Multiple comparison procedures, Wiley, New York.

[8] Navin, F. P. D. and Wheeler, R. J. (1969). "Pedestrian flow characteristics." Traffic Eng., 39(4), 30-36.

[9] Park, B. B., Won, J. and Yun, I. (2006). "Application of microscopic simulation model calibration and validation procedure: Case study of coordinated actuated signal system." Transportation Research Record: Journal of the Transportation Research Board, No. 1978, 113-122. 